\title{
AGE PROBLEM BASED ON THE ABUNDANCE RATIO FROM A HIGH-REDSHIFT QSO
}

\author{
T. TSUJIMOTO \\ National Astronomical Observatory, Mitaka, Tokyo 181-8588, \\ Japan \\ AND \\ Y. YOSHII, K. KAWARA \\ Institute of Astronomy, Faculty of Science, University of Tokyo, \\ Mitaka, Tokyo 181-8588, Japan
}

\begin{abstract}
The age of a high-redshift QSO can constrain the cosmology by placing a limit on the cosmological time. We use the observed $\mathrm{Fe}_{\mathrm{II}}$ (UV+optical) $/ \mathrm{Mg}_{\text {II }} \lambda 2798$ flux ratio from the broad-line gas of a QSO at $z=3.62$ to estimate the abundance ratio $[\mathrm{Mg} / \mathrm{Fe}]$ and predict that the QSO is at least $1.3 \mathrm{Gyr}$ old from the chemical analysis using the abundance ratio $[\mathrm{Mg} / \mathrm{Fe}]$ as a cosmic clock. Our result favors a nonzero cosmological constant and thus conflicts with a flat, matter-dominated universe.
\end{abstract}

\section{Introduction}

Type II supernovae (SNe II) contribute to the heavy element production in the early chemical evolution of galaxies. Type Ia supernovae (SNe Ia), in contrast, produce heavy elements with a considerably longer timescale in the late phases of the galactic evolution, as they are due to thermonuclear explosions of accreting white dwarfs. The yields of SNe II and SNe Ia and thus their nucleosynthesis roles in the chemical evolution of galaxies are distinctly different, i.e., SNe Ia eject much larger amount of $\mathrm{Fe}$ than $\mathrm{SNe}$ II, but have negligible contributions to the $\mathrm{O}$ and $\mathrm{Mg}$ abundances (see Tsujimoto et al. 1995). These differencies between SNe II and SNe Ia enable us to measure the age of galaxies (QSOs) by using the abundance ratios of $\mathrm{O} / \mathrm{Fe}$ or $\mathrm{Mg} / \mathrm{Fe}$ as cosmic clocks.

Here we determine the lower limit on an age of QSO B1422+231 at $z=3.62$ from the abundance ratio $[\mathrm{Mg} / \mathrm{Fe}]$, which is estimated from the observed flux ratio $\mathrm{Fe}_{\mathrm{II}}(\mathrm{UV}+$ optical $\left.)\right] / \mathrm{Mg}_{\mathrm{II}} \lambda 2798$ obtained by Kawara et 
al. (1996). The derived age puts the powerful constraint on cosmology, suggesting that there exits the so-called age problem at $z=3.62$ as well as at $z=0$ and 1.55 .

\section{Age Problems at $z=0,1.55$}

In this section we mention the present status of the classical age problem based on the Galactic globular cluster age and another age problem resulting from a recent discovery of a 3.5-Gyr-old galaxy at $z=1.55$.

\subsection{AGE PROBLEM AT $Z=0$}

Ages of Galactic globular clusters (GCs) give the lower limit on the age of the universe. The age of a GC can be deduced from stellar evolution calculations and is estimated to be about $15 \mathrm{Gyr}$ (e.g., 15.8 2 2.1 Gyr by Bolte \& Hogan 1995; $14.56 \pm 2.49$ Gyr by Chaboyer et al. 1996). On the other hand, the standard cosmological model of a flat, matter-dominated universe with the Hubble constant $H_{0}=60-80 \mathrm{~km} \mathrm{~s}^{-1} \mathrm{Mpc}^{-1}$ estimated from almost all observations implies a cosmic age of 8-11 Gyr, which is inconsistent with the ages of Galactic GCs. To solve this discrepancy, we should adopt the density of the universe $\Omega<0.2$, or introduce the cosmological constant $\Lambda$ if the estimated ages of GCs are correct. Recently, a possibility of younger age of GCs has been discussed since the preference of the bright absolute magnitude of RR Lyrae stars $M_{V}(\mathrm{RR})$ was inferred from the Hipparcos measurements of parallaxes of Cepheids (Feast \& Catchpole 1997) and subdwarfs (Reid 1997). However, Tsujimoto, Miyamoto, \& Yoshii (1997) present the direct determination of $M_{V}(\mathrm{RR})$ from statistical treatments of Hipparcos proper motions and trigonometric parallaxes of RR Lyraes and have confirmed the faint $M_{V}(\mathrm{RR})$, thus suggesting that the so-called age problem would not be resolved or alleviated yet.

Moreover, a simple, independent estimate of the age of one very old star (CS22892-052), using a method called nucleocosmochronology, is presented by Sneden et al. (1996). From the deficiency in a radio-active $r$-process element $\mathrm{Th}$, they derive the age of $15.2 \pm 3.7 \mathrm{Gyr}$, which is in good agreement with the ages of GCs.

\subsection{AGE PROBLEM AT $Z=1.55$}

Dunlop et al. (1996) discoverd a red radio galaxy LBDS 53W091 at $z=1.55$. By fitting the observed spectrum to stellar population models and taking advantage of the age-sensitivity of the near-ultraviolet spectrum, Spinrad et al. (1997) derive a minimum age of $3.5 \mathrm{Gyr}$. On the other hand, the Universe 
is only $2-2.6$ Gyr old at $z=1.55$ for a flat, matter-dominated universe. This is the second age problem.

\section{Age Problem at $z=3.62$}

The rest-frame UV-optical spectrum of the gravitational lensed QSOB1422 +231 at $z=3.62$ is presented by Kawara et al. (1996). Their observed $\mathrm{Fe}_{\text {II }}$ (UV+optical) $/ \mathrm{Mg}_{\text {II }} \lambda 2798$ flux ratio is $12.2 \pm 3$.9. Similar flux ratios $\sim 8$ are obtained for QSOs at low and intermediate redshifts (Wills, Netzer, \& Wills 1985). Wills et al. also calculate the strengths of the emission lines by their photoionization models and conclude that the observed flux ratio $\sim 8$ suggests an overabundance of Fe by a factor of $\sim 3$ relative to $\mathrm{Mg}$. Therefore the lower bound for the observed flux ratio from B1422+231 corresponds to the abundance ratio $[\mathrm{Mg} / \mathrm{Fe}] \sim-0.5$. It means that $\mathrm{B} 1422+231$ has the abundance ratio $[\mathrm{Mg} / \mathrm{Fe}] \lesssim-0.5$.

It is noted that the abundance ratio $[\mathrm{Mg} / \mathrm{Fe}]$ in $\mathrm{B} 1422+231$ is quite different from those in the solar neighborhood stars. The negative ratio implies a significant contribution of $\mathrm{Fe}$ from $\mathrm{SNe}$ Ia. Such a large contribution from SNe Ia to the broad-line gas is possible if QSOs are associated with vigorous star formation. This effect is understood as follows. The heavy elements ejected from SNe II during the burst are largely trapped in stars and a small fraction of the heavy elements is contained in the gas. On the other hand, SNe Ia start to occur after the burst, then the heavy elements ejected from $\mathrm{SNe}$ Ia are restored in the gas because of the absence of star formation. Therefore after the burst, the gas becomes considerably enriched with $\mathrm{Fe}$ from $\mathrm{SNe}$ Ia.

Hamann \& Ferland $(1992,1993)$ construct the chemical evolution models for QSOs so as to explain the $\mathrm{N}_{\mathrm{V}} / \mathrm{C}_{\mathrm{IV}}$ line ratios at high- $z$ QSOs. They conclude that high- $z$ QSOs were enriched with heavy elements in a burst of star formation, which is consistent with an above implication of $[\mathrm{Mg} / \mathrm{Fe}]$ in B1422+231. They derive enhanced star formation rates for relatively low-metallicty QSOs $(\nu=5.0)$, high-metallicty QSOs $(\nu=6.7)$, and extreme high-metallicity QSOs $(\nu=7.6)$, respectively [ $\nu$ : the coefficient of star formation rate; $\nu=0.27$ for their solar neighborhood model]. The star formation rate for extreme high-metallicity QSOs gives the shortest timescale for the enrichment by heavy elements in QSOs.

The lifetime of SN Ia progenitors as well as the star formation rate is a critical parameter for calculating the time evolution of Fe. The lifetime $t_{\text {Ia }}$ of SN Ia progenitors is deduced from the chemical evolution in the solar neighborhood (Yoshii, Tsujimoto, \& Nomoto 1996). It is found that the range of $t_{\mathrm{Ia}}$ is strictly confined within $0.5-3 \mathrm{Gyr}$.

Using the above determined parameters together with a Salpeter initial 

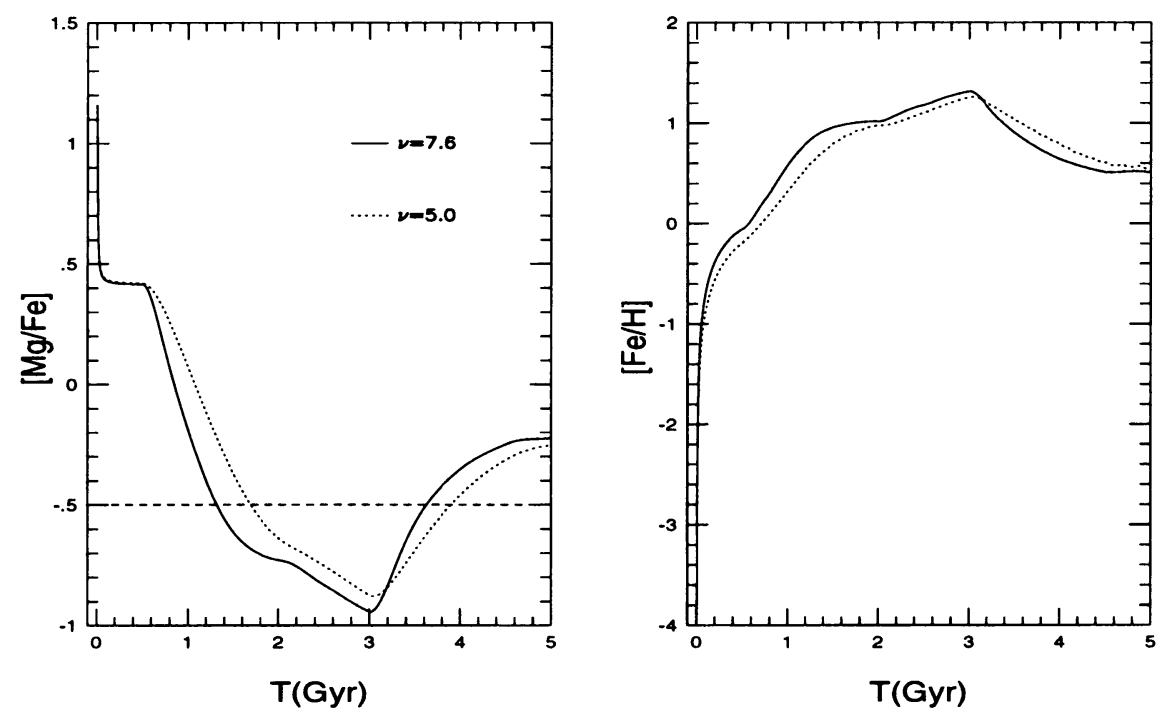

Figure 1. The evolutionary change in $[\mathrm{Mg} / \mathrm{Fe}]$ and $[\mathrm{Fe} / \mathrm{H}]$ predicted by the models with high star formation rates.

mass function (IMF), we calculate the evolutionary change in $[\mathrm{Mg} / \mathrm{Fe}]$ and $[\mathrm{Fe} / \mathrm{H}]$ in QSOs (Figure 1). The dashed line denotes the upper bound for the observed $[\mathrm{Mg} / \mathrm{Fe}]$. The intersection between the theoretical curve of $[\mathrm{Mg} / \mathrm{Fe}]$ predicted by the model with the extreme high star formation rate and the observed upper bound corresponds to the strong lower limit on the age of the QSO B1422+231. We therefore obtain $T_{\mathrm{QSO}}$ at $\mathrm{z}=3.62 \geq 1.3 \mathrm{Gyr}$, whereas the Universe is only $0.6-0.8$ Gyr old at $z=3.62$ for a flat, matterdominated universe.

Figure 2 shows the predicted age-redshift relations for three cosmological models, i.e., a flat, matter-dominated universe $(\Omega=1, \Lambda=0)$, an open universe $(\Omega=0.2, \Lambda=0)$, and a cosmological constant-dominated universe $(\Omega=0.2, \Lambda=0.8)$, together with three age constraints. The solid lines for each panel denote the cases for $H_{0}=60$ and $80 \mathrm{~km} \mathrm{~s}^{-1} \mathrm{Mpc}^{-1}$, assuming the formation epoch at $z=10$. The case for the formation epoch at $z=5$ with $H_{0}=60 \mathrm{~km} \mathrm{~s}^{-1} \mathrm{Mpc}^{-1}$ is also shown by the dashed line.

\section{Conclusion}

A flat, matter-dominated universe conflicts with age problems at three different redshifts, i.e., $z=0,1.55$ and 3.62. The age constraint from a high- $z$ QSO is more stringent than those from the Galactic globular cluster or a galaxy at $z=1.55$. An open universe $(\Omega=0.2)$ is acceptable if we adopt $H_{0}$ 

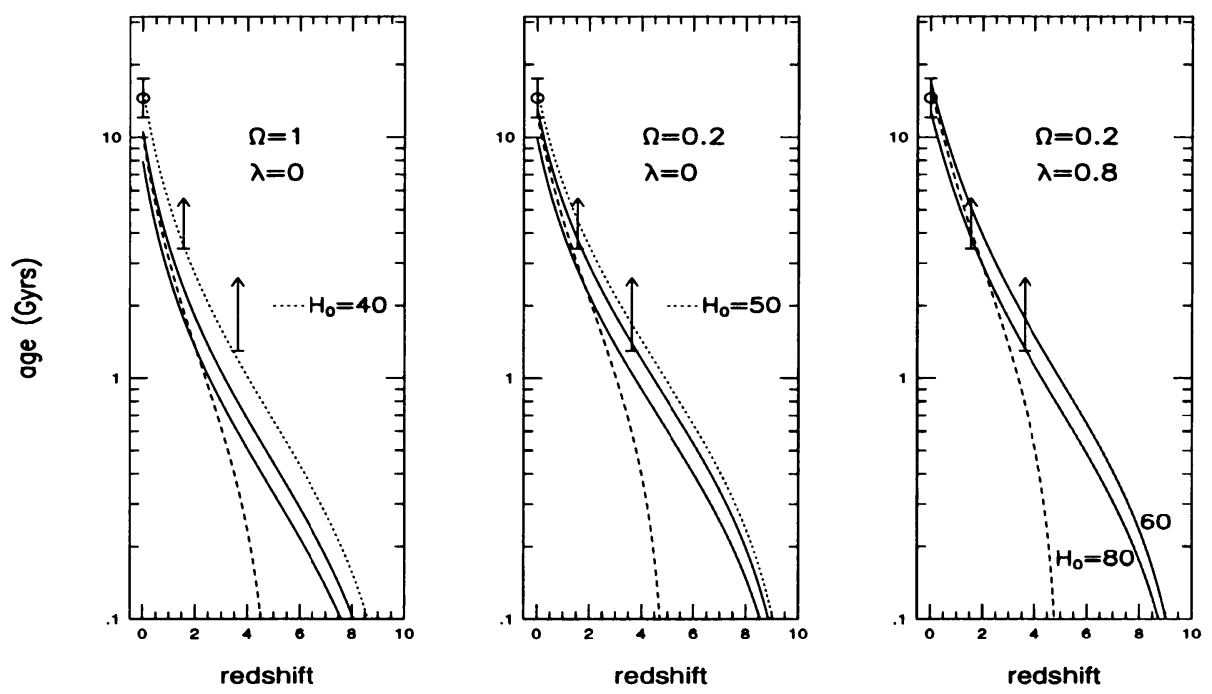

Figure 2. The age-redshift relations for three cosmological models.

less than $65 \mathrm{~km} \mathrm{~s}^{-1} \mathrm{Mpc}^{-1}$. However a preferable age constraint at $z=3.62$ inferred from the observed $\mathrm{Fe}_{\text {II }}$ (UV+optical) $/ \mathrm{Mg}_{\text {II }} \lambda 2798$ flux ratio $\sim 12$ excludes a open universe. This age problem is more serious if the IMF in QSOs is weighted toward forming more massive stars than in the solar neighborhood. It is likely that in the starburst the IMF has a shallower slope or has a truncation at a large lower stellar mass limit. In this case the calculated $[\mathrm{Mg} / \mathrm{Fe}]$ becomes higher and it results in giving the older age for the QSO. On the other hand, this age constraint is consistent with the cosmological time predicted by a cosmological constant-dominated universe.

\section{References}

Bolte, M. and Hogan, C.J. 1995, Nature, 376, 399

Chaboyer, B., Demarque, P., Kernan, P.J. and Krauss, L.M. 1996, Science, 271, 957

Dunlop, J. et al. 1996, Nature, 381, 581

Feast, M.W. and Catchpole, R.W. 1997, M.N.R.A.S., 286, L1

Hamann, F. and Ferland, G. 1993, Ap.J., 418, 11

Hamann, F. and Ferland, G. 1992, Ap.J., 391, L53

Kawara, K., Murayama, T., Taniguchi, Y. and Arimoto, N. 1996, Ap.J., 470, L85

Ried, I.N. 1997, A.J., 114, 161

Sneden, C. et al. 1996, Ap.J., 467, 819

Spinrad, H. et al. 1997, Ap.J., 484, 581

Tsujimoto, T., Miyamoto, M. and Yoshii, Y. 1997, Ap.J.Lett, in press

Tsujimoto, T. et al. 1995, M.N.R.A.S., 277, 945

Wills, B.J., Netzer, H. and Wills, D. 1985, Ap.J., 288, 94

Yoshii, Y., Tsujimoto, T. and Nomoto, K. 1996, Ap.J., 462, 266 